This is a self-archived - parallel published version of this article in the publication archive of the University of Vaasa. It might differ from the original.

\title{
Spatial Modulation or Spatial Multiplexing for mmWave Communications?
}

Author(s): Elkawafi, Salma; Younis, Abdelhamid; Mesleh, Raed; Abouda, Abdulla; Elbarsha, Ahmed; Elmusrati, Mohammed

Title: $\quad$ Spatial Modulation or Spatial Multiplexing for mmWave Communications?

Year: $\quad 2019$

Version: Accepted manuscript

Copyright (C) 2019 Springer.

Please cite the original version:

Elkawafi, S., Younis, A., Mesleh, R., Abouda, A., Elbarsha, A. \& Elmusrati, M. (2019). Spatial Modulation or Spatial Multiplexing for mmWave Communications?. In: Sucasas V., Mantas G. \& Althunibat S. (eds.) Broadband Communications, Networks, and Systems. BROADNETS 2018, 237-246. Lecture Notes of the Institute for Computer Sciences, Social Informatics and Telecommunications Engineering, vol. 263. Cham: Springer. https://doi.org/10.1007/978-3030-05195-2_23 


\title{
Spatial Modulation or Spatial Multiplexing for mmWave Communications?
}

\author{
Salma Elkawafi ${ }^{1}$, Abdelhamid Younis ${ }^{1}$, Raed Mesleh ${ }^{2(凶)}$, Abdulla Abouda ${ }^{3}$, \\ Ahmed Elbarsha ${ }^{1}$, and Mohammed Elmusrati ${ }^{4}$ \\ 1 Electrical and Electronics Engineering Department, Faculty of Engineering, \\ University of Benghazi, P.O. Box 7051, Benghazi, Libya \\ \{salma.elkawafi, a.alhassi, ahmed.elbarsha\}@uob.edu.ly \\ 2 Electrical and Communications Engineering Department, School of Electrical \\ Engineering and Information Technology, German Jordanian University, \\ P.O. Box 35247, Amman 11180, Jordan \\ raed.mesleh@gju.edu.jo \\ 3 Almadar Research and Development Office, Almadar Aljadid Company, \\ Tripoli, Libya \\ a. abouda@almadar.ly \\ 4 The Department of Computer Science, University of Vaasa, Vaasa, Finland \\ moel@uwasa.fi
}

\begin{abstract}
In this paper, two large scale (LS)-multiple-input multipleoutput (MIMO) systems and their performance over 3D statistical outdoor millimeter wave (mmWave) channel model are considered and thoroughly analyzed. Namely, spatial multiplexing (SMX) and spatial modulation (SM) systems are considered. The performance of both systems in terms of average bit error ratio (ABER) and channel capacity are derived and studied. Obtained results divulge that SM can achieve higher theoretical capacity than SMX system. Further, SMX system is shown to offer better ABER and mutual information performance as compared to SM system for the same system configuration. Yet, SM demonstrate significant energy efficiency (EE) enhancement for large scale number of transmit antennas.
\end{abstract}

Keywords: Millimeter-wave (mmWave) communication Spatial modulation (SM) - Spatial Multiplexing (SMX) Large-scale MIMO (LS-MIMO)

\section{Introduction}

CISCO anticipated recently [8] that wireless mobile data traffic will witness tremendous growth in the coming few years. Such growth is impelled by the huge spread of IoT applications and video streaming. It is anticipated that data

Granted by Almadar Research and Development Office.

(C) ICST Institute for Computer Sciences, Social Informatics and Telecommunications Engineering 2019

Published by Springer Nature Switzerland AG 2019. All Rights Reserved

V. Sucasas et al. (Eds.): BROADNETS 2018, LNICST 263, pp. 237-246, 2019.

https://doi.org/10.1007/978-3-030-05195-2_23 
Table 1. Spatial Multiplexing and Spatial Modulation

\begin{tabular}{l|l|l}
\hline & SMX & SM \\
\hline $\begin{array}{l}\text { Spectral } \\
\text { Efficiency }\end{array}$ & $\begin{array}{l}\text { The spectral efficiency increases } \\
\text { linearly with the number of } \\
\text { transmit antennas }\end{array}$ & $\begin{array}{l}\text { The spectral efficiency increases } \\
\text { by base two logarithm of the } \\
\text { number of transmit antennas }\end{array}$ \\
\hline $\begin{array}{l}\text { Computational } \\
\text { Complexity }\end{array}$ & $\begin{array}{l}\text { The CC increases at the receiver } \\
\text { since it has to resolve the } \\
\text { inter-channel interference (ICI) } \\
\text { imposed by transmitting } \\
\text { simultaneously from all } \\
\text { antennas }\end{array}$ & $\begin{array}{l}\text { It is notfected by ICI and } \\
\text { provides the same } \\
\text { computational complexity of a } \\
\text { single transmit antenna systems }\end{array}$ \\
\hline $\begin{array}{l}\text { Hardware } \\
\text { Complexity }\end{array}$ & $\begin{array}{l}\text { All transmit antennas are active } \\
\text { and each antenna requires } \\
\text { individual RF chain, which } \\
\text { complicate the deployment and } \\
\text { increase the cost [12] }\end{array}$ & $\begin{array}{l}\text { Only one transmit antenna is } \\
\text { active on each time so only one } \\
\text { RF chain is required which } \\
\text { reduces the hardware } \\
\text { complexity }\end{array}$ \\
\hline
\end{tabular}

traffic will reach 30.6 Exabytes per month by 2020. Yet, the existing spectrum is overcrowded and can not accommodate such massive increase in data rates [8]. Therefore, interest in utilizing unregulated wide spectrum has increased in the past few years.

One of the promising technologies for future $5 \mathrm{G}$ and beyond wireless systems is millimetre-wave (mmWave) communication [16], which utilizes huge range of unused spectrum and promises significant enhancement is spectral efficiency. Another technique that witness huge research interest is large scale (LS)multiple-input multiple-output (MIMO) [3] systems, which leads to very high data rates. Combining mmWave with LS-MIMO promises to achieve the needed capacity and to accommodate the demand for high-data rate wireless systems.

mmWave technology provides several gigahertz bandwidths and is a promising solution for the spectrum congestion in current wireless standards. mmWave offers a bountiful spectrum spanning from $24-300 \mathrm{GHz}$ that can be employed to achieve multi-gigabits per second data rates. Hence, it addresses many challenges in future wireless systems including very high data rates, and real-time and reliable communications [6]. Yet, the propagation of mmWave signals require accurate channel modeling that attracted significant research interest in literature $[1,16]$. Among the many existing models, a 3D channel model is shown in [16] to be the most comprehensive and accurately match measurement data. Therefore, it is considered in this study.

Developing an efficient MIMO technique is an active research topic for the past 20 years aiming to boost the capacity of wireless systems [19]. As such, MIMO systems are the main technology in $4 \mathrm{G}$ wireless standard and will play a major role in $5 \mathrm{G}$ and beyond standards [13]. Yet, practical implementation of MIMO systems face several challenges and two promising technologies are widely studied. Namely, spatial multiplexing (SMX) [9] and spatial 
modulation (SM) $[2,7,11]$ MIMO systems promise significant advantages and undergoes intensive research interest. The advantages and disadvantages for each system are tabulated in Table 1 . The requirement for massive data rate growth in future systems will rely on LS-MIMO systems [19]. Deploying large number of transmit antennas promises much higher spectral efficiency than conventional MIMO schemes [19]. Combining LS-MIMO and mmWave systems assure significant performance enhancement in terms of spectral efficiency and bandwidth, and are the key technologies for future $5 \mathrm{G}$ wireless systems.

This study aims at highlighting the performance of SMX and SM for mmWave system by evaluating their average bit error ratio (ABER), capacity and energy efficiency (EE). Interesting results are reported where it is revealed that SMX is superior to SM in terms of ABER assuming similar MIMO configuration. However, considering LS MIMO system significantly ameliorate SM performance. Even though this enhancement requires large number of antennas, they can be deployed at marginal cost of SM as discussed in [12]. Also, the channel capacity of SM is shown to be higher than that of SMX. Thereby, both MIMO schemes have several pros and cons when combined with mmWave communication. The use of which system depends on the data rate and ABER. For LS-MIMO with hundred of antennas, SM is preferred with its much cheaper energy implementation. Whereas SMX is better scheme for small scale MIMO setup since it promises lower ABER performance.

The rest of the paper is organized as follows: Sect. 2 introduces the system and channel models. The mutual information and theoretical capacity for SMX and SM systems are derived and discussed in Sect. 3. Section 4 summarizes the obtained results. Finally, the paper is concluded in Sect. 5.

\section{System and Channel Models}

\subsection{MIMO Systems}

SMX: In SMX, all transmit antennas are activated simultaneously to transmit $\eta=N_{t} \log _{2}(M)$ bits, with each antenna transmitting $M$-quadrature amplitude modulation (QAM) symbol [9]. Activating all antennas simultaneously require that they should be synchronized and the overall transmit power is divided among them.

SM: In SM, only one transmit antenna is activated each time instance. Thus, the spectral efficiency of SM is $\eta=\log _{2} N_{t}+\log _{2} M$ bits [5,11]. In SM the incoming $\eta$ data bits are divided into two parts;

i. The first part, $\log _{2}\left(N_{t}\right)$, bits determine which transmit antenna $l_{t}$ is active, where $l_{t}=1,2, . ., N_{t}$.

ii. The second part with $\log _{2} M$ bits modulate a symbol driven from $M$-QAM constellation and then transmitted from the $l_{t}$ transmit antenna. 
The generated SM unique symbol vector, $\mathbf{x}_{t}$, contains only single nonzero element and the vector is broadcasted over a mmWave MIMO channel matrix with an $N_{r} \times N_{t}$ dimension and a transfer function of $\mathbf{H}$. The signal at the input of the receive antennas experiences an $N_{r}$-dim additive white Gaussian noise (AWGN) $(\mathbf{n})$, with zero mean and $\sigma_{n}^{2}$ variance.

The signal at the receiver can be written as

$$
\mathbf{y}=\mathbf{H} \mathbf{x}_{t}+\mathbf{n}
$$

Considering that the transmitted symbol is normalized, i.e., $E_{s}=\mathrm{E}\left[\|\mathbf{H x}\|_{\mathrm{F}}^{2}\right]=$ $N_{r}$, the signal-to-noise-ratio (SNR) is written as $\mathrm{SNR}=E_{s} / N_{0}=1 / \sigma_{n}^{2}$, with $\|\cdot\|_{\mathrm{F}}$ being the Frobenius norm.

\subsection{D Statistical mmWave Channel Model}

Assuming that all transmit antennas are omni-directional antennas and operating at a mmWave frequency. The channel between the $n_{t}$-th and $n_{r}$-th transmit and receive antennas channel, denoted as $h_{n_{t}, n_{r}}(t)$, is written as [15],

$$
\underset{n_{t}, n_{r}}{h(t)}=\sum_{l=1}^{L} \underset{n_{t}, n_{r}}{h^{l}} a_{l} e^{j \varphi_{l}} \delta\left(t-\tau_{l}\right) \delta\left(\Theta-\underset{n_{t}, l}{\Theta}\right) \delta\left(\Phi-\underset{n_{r}, l}{\Phi}\right)
$$

with $\underset{n_{t}, n_{r}}{h^{l}}$ denoting the complex channel fading of the $l$-th sub-path, among the existing $L$ multi-path components, between the $n_{t}$-th and $n_{r}$-th antennas, and the amplitude, phase and propagation-delay of the same sub-path are denoted by $a_{l}, \varphi_{l}$ and $\tau_{l}$. Also, the angle of departure (AOD) and angle of arrival (AOA) azimuth/elevation angle vectors are given by $\Theta_{n_{t}, l}$ and $\Phi_{n_{r}, l}$ for the $n_{t}$-th and $n_{r}$-th antennas. Assuming that the antennas at both side are aligned along the $z$-dimension and separated by equidistant $d$, the channel in (2) can be simplified to,

$$
\left.\begin{array}{c}
h(t)=\sum_{n_{t}, n_{r}}^{L} \underset{l=1}{h_{t}, n_{r}} a_{l} e^{j \varphi_{l}} \delta\left(t-\tau_{l}\right) \delta\left(\theta^{z}-\theta_{n_{t}, l}^{z}\right.
\end{array}\right) \delta\left(\begin{array}{c}
\phi^{z}-\underset{n_{t}, l}{\phi^{z}}
\end{array}\right)
$$

with the elevation AOD and AOA for the $n_{t}$-th and $n_{r}$-th transmit and receive antennas are respectively denoted by $\theta_{n_{t}, l}^{z}$ and $\phi_{n_{r}, l}^{z}$.

According to [14], the transfer function in (3) is

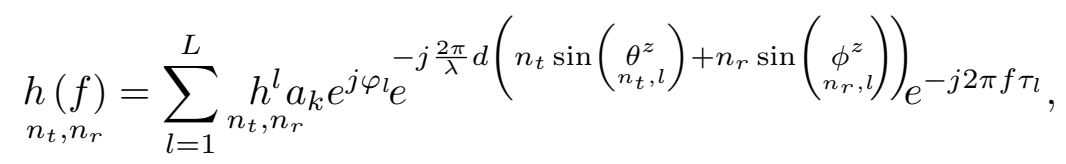

with $\lambda$ being the wavelength.

The parameters in this study, $a_{l}, \varphi_{l}, \theta_{n_{t}, l}^{z}, \phi_{n_{r}, l}^{z}$, and $\tau_{l}$, are generated according to [16] considering outdoor mmWave model. 


\subsection{Optimum Receiver}

The optimum decoder is considered for both SMX and SM in this paper, which can be written as

$$
\hat{\mathbf{x}}_{t}=\underset{\mathbf{x} \in \mathbf{X}}{\arg \min }\left\{\|\mathbf{y}-\tilde{\mathbf{H}} \mathbf{x}\|_{\mathrm{F}}^{2}\right\}
$$

with all possible transmitted vector for each system and grouped in the set $\mathbf{X}$ contains every possible $\left(N_{t} \times 1\right)$ and the estimated vector is denoted by $\hat{\imath}$.

\section{Capacity Analysis}

\subsection{Mutual Information}

To compute the capacity of SM and SMX systems, the mutual information, which represents the number of received and decoded bits without errors, should be computed. For SMX, $I(\mathbf{X} ; \mathbf{Y})$, is given by [10],

$$
I(\mathbf{X} ; \mathbf{Y})=\mathrm{E}_{\mathbf{H}}\{I(\mathbf{X} ; \mathbf{Y} \mid \mathbf{H})\}=\mathrm{E}_{\mathbf{H}}\{H(\mathbf{Y} \mid \mathbf{H})-H(\mathbf{Y} \mid \mathbf{X}, \mathbf{H})\}
$$

where $H(\cdot)$ denoting the entropy function and all possible $\left(N_{r} \times 1\right)$ receive vectors are grouped in $\mathbf{Y}$. Then, $I(\mathbf{X} ; \mathbf{X} \mid \mathbf{H})$ is written as

$$
I(\mathbf{X} ; \mathbf{Y} \mid \mathbf{H})=\eta-N_{r} \log _{2}(e)-\mathrm{E}_{\mathbf{Y}}\left\{\log _{2} \sum_{\mathbf{x} \in \mathbf{X}} e^{\frac{-\|\mathbf{y}-\mathbf{H} \times\|_{\mathrm{F}}^{2}}{\sigma_{n}^{2}}}\right\} .
$$

In SM, the channel paths are used as a spatial constellation symbols that are modulated by source data bits and used to convey part of the information data. Hence, the mutual information for SM systems is given by,

$$
\begin{aligned}
I(\mathcal{H}, \mathcal{S} ; \mathbf{Y}) & =H(\mathbf{Y})-H(\mathbf{Y} \mid \mathcal{H}, \mathcal{S}) \\
& =\eta-N_{r} \log _{2}(e)-\mathrm{E}_{\mathbf{Y}}\left\{\log _{2} \sum_{\substack{\mathcal{H}_{\ell} \in \mathcal{H} \\
\mathcal{S}_{\imath} \in \mathcal{S}}} e^{\frac{-\left\|\mathbf{y}-\mathcal{H}_{\ell} \mathcal{S}_{\imath}\right\|_{\mathrm{F}}^{2}}{\sigma_{n}^{2}}}\right\},
\end{aligned}
$$

\subsection{Capacity}

Spatial Multiplexing: By definition, the capacity is [10],

$$
C=\max _{p_{\mathbf{X}}} I(\mathbf{X} ; \mathbf{Y} \mid \mathbf{H}),
$$

where the theoretical capacity is achieved maximization by selecting proper $p_{\mathbf{X}}$ such that the mutual information is maximized, with $p_{\mathbf{X}}$ being the probability distribution function (PDF) of the transmitted space of vectors $\mathbf{X}$. 
Substituting (6) in (10), the capacity for SMX is rewritten as,

$$
C=\max _{p_{\mathbf{X}}}(H(\mathbf{Y} \mid \mathbf{H})-H(\mathbf{Y} \mid \mathbf{X}, \mathbf{H})) .
$$

A significant conclusion can be drawn from (11) where the entropy $H(\mathbf{Y} \mid \mathbf{X}, \mathbf{H})$ is not function of $\mathbf{X}$. Thereby, (11) is maximized if $H(\mathbf{Y} \mid \mathbf{H})$ is maximized. Considering that the entropy is maximized if a zero-mean complex Gaussian distribution is considered as derived in [17]. As such, the maximum entropy of $\mathbf{Y}$ is,

$$
H(\mathbf{Y} \mid \mathbf{H})=N_{r} \log _{2}\left(\pi e\left(\frac{1}{N_{t}} \mathbf{H} \mathbf{H}^{H}+\sigma_{n}^{2} \mathbf{I}_{N_{r}}\right)\right)
$$

The entropy of $\mathbf{Y}$ knowing $\mathbf{X}$ is,

$$
H(\mathbf{Y} \mid \mathbf{X}, \mathbf{H})=N_{r} \log _{2}\left(\pi \sigma_{n}^{2} e\right) .
$$

With the help of (11)-(13), the ergodic capacity of SMX is given by,

$$
C_{\text {ergodic }}=\mathrm{E}_{\mathbf{H}}\left\{\log _{2}\left(\left|\mathbf{I}_{N_{r}}+\frac{1}{\sigma_{n}^{2} N_{t}} \mathbf{H H}^{H}\right|\right)\right\}
$$

Spatial Modulation: In SM the information bits are modulated in the different constellations symbols and channel vectors. Therefore, the capacity in (10) can be re-written as,

$$
C=\max _{p_{\mathcal{H}}, p_{\mathcal{S}}} I(\mathcal{H}, \mathcal{S} ; \mathbf{Y})=\max _{p_{\mathcal{H}}, p_{\mathcal{S}}}\{H(\mathbf{Y})-H(\mathbf{Y} \mid \mathcal{H}, \mathcal{S})\}
$$

where $p_{\mathcal{H}}$ and $p_{\mathcal{S}}$ are the PDFs of $\mathcal{H}$ and $\mathcal{S}$ respectively.

As in (11), the left hand size of (15) does not depend on $\mathcal{S}$ nor $\mathcal{H}$. Thus, the maximization in (15) is only of $H(\mathbf{Y})$. The entropy $H(\mathbf{Y})$ is maximized when $\mathbf{Y} \sim \mathcal{C N}\left(\mathbf{0}_{N_{r}}, \sigma_{Y}^{2} \mathbf{I}_{N_{r}}\right)$, with $\sigma_{\mathbf{Y}}^{2}$ denoting the variance of $\mathbf{Y}$ and $\mathbf{0}_{N_{r}}$ is an $N_{r}$-length all zeros vector. From (1), the received signal is complex Gaussian distributed only if $\mathcal{H S} \sim \mathcal{C N}\left(\mathbf{0}_{N_{r}}, \mathbf{I}_{N_{r}}\right)$, where $\mathbf{0}_{N}$ is an $N$-length all zeros vector, and $\mathbf{I}_{N}$ is an $N \times N$ identity matrix. By assuming $\mathcal{H} \mathcal{S}$ is complex Gaussian distributed, the entropy of $\mathbf{Y}$ following the same steps as discussed for (13) is,

$$
H(\mathbf{Y})=N_{r} \log _{2}\left(\pi e\left(1+\sigma_{n}^{2}\right)\right) .
$$

Under these conditions and with the help of (11), (13), and (16), the space modulation techniques (SMT) capacity is given by,

$$
C_{\text {ergodic }}=N_{r} \log _{2}\left(1+1 / \sigma_{n}^{2}\right)=N_{r} \log _{2}(1+\mathrm{SNR}) .
$$

\section{Results}

Presented results in this section, Figs. 1, 2 and 3, study and compare the performance of SM and SMX over 3D mmWave statistical channel model while varying different system and channel parameters. It is assumed that a basestation with $N_{t}$ transmit antennas communicates with a single user that has $N_{r}$ receive antennas. 


\subsection{ABER Performance Comparison}

Figure 1 depicts the ABER while varying $N_{t}$ for both SMX and SM systems while achieving a spectral efficiency of $\eta=16$ and considering $N_{r}=2$. Reported results reveal that SMX outperforms SM by about $3 \mathrm{~dB}$ in SNR. This can be attributed to the fact that SMX requires smaller constellation diagram than SM to achieve the target spectral efficiency.

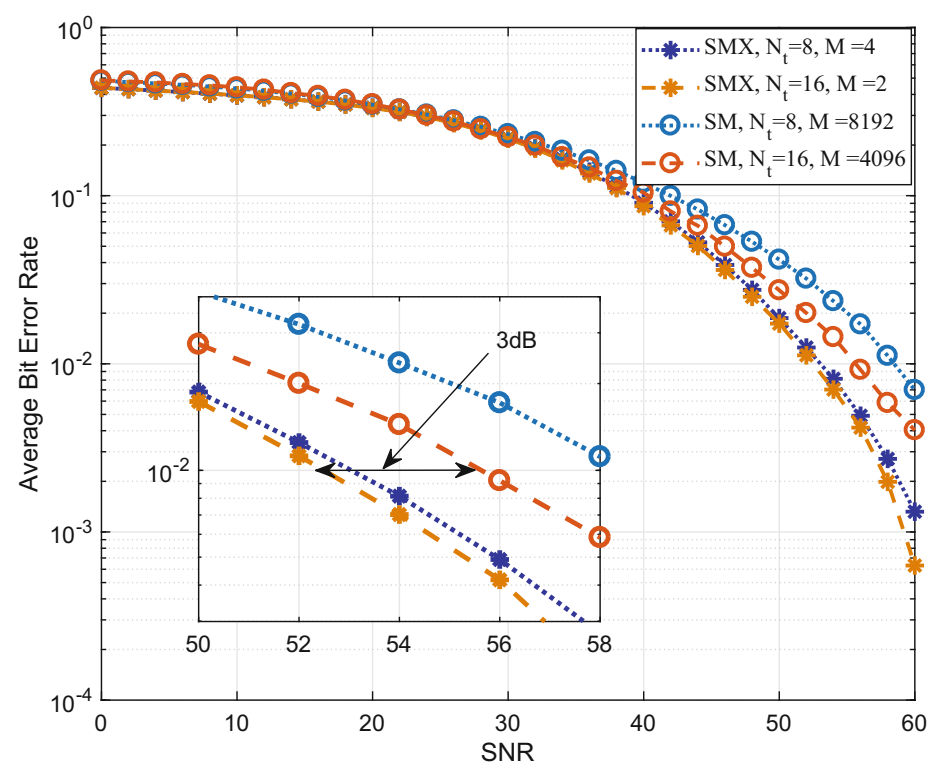

Fig. 1. ABER performance of SM and SMX assuming $N_{t}=8$, and 16, $N_{r}=2$ and a spectral efficiency of $\eta=16 \mathrm{bps} / \mathrm{Hz}$.

Considering the case where $N_{t}=16$ while varying $N_{r}$ for the same $\eta=16$ is studied and results are illustrated in Fig. 2. Increasing the number of receive antennas enhances the performance of SMX and SM systems. Yet, SMX still outperforms SM by about 6 , and $11 \mathrm{~dB}$ respectively for $N_{r}=2$ and 4 . This behavior can be attributed to the same reason as discussed earlier.

\subsection{Capacity Results}

Mutual information results are depicted in Fig. 3 for $\eta=8$ and 16 while assuming $N_{t}=8$ and $N_{r}=4$ antennas. The capacity curves for both systems are also depicted. It is observed that SM and SMX perform nearly the same at a spectral efficiencies of 8 . Yet, for $\eta=16$ bits, SMX offers higher mutual information than $\mathrm{SM}$. It can be seen that at $\mathrm{SNR}=30 \mathrm{~dB}$, SMX mutual information is 3.32 bits higher than the mutual information of SM. Even though SMX outperforms SM it term of mutual information, SM can achieve higher capacity as can be seen from the figure, where for SMX it is required more $11 \mathrm{~dB}$ to achieve the same spectral efficiency, 16 bits. 


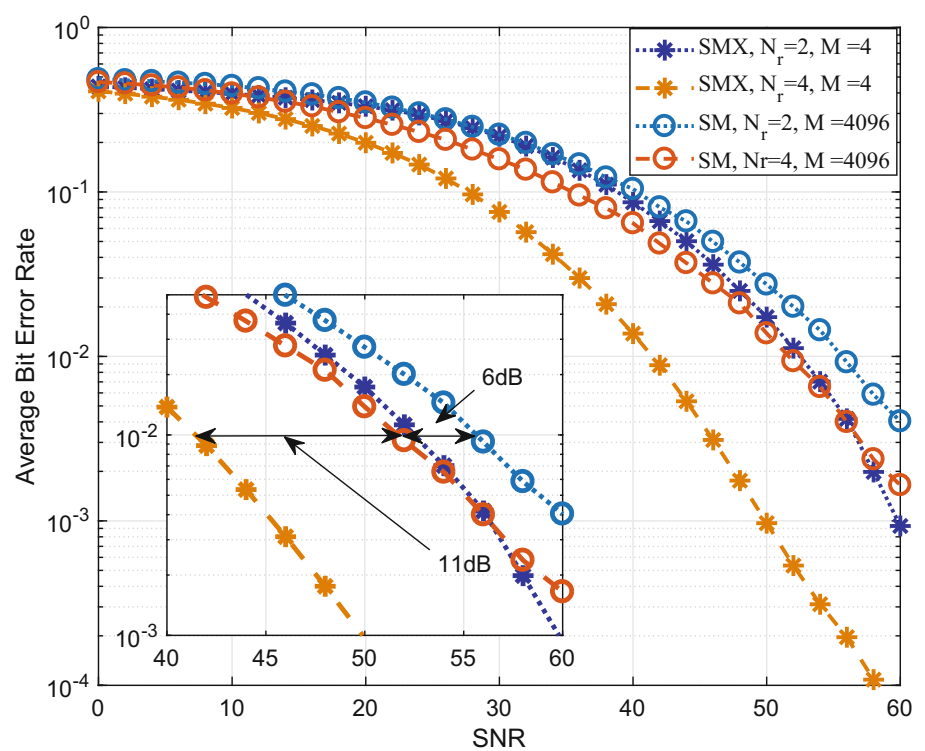

Fig. 2. ABER performance of SM and SMX assuming $N_{t}=16, N_{r}=2$ and 4 and a spectral efficiency of $\eta=16 \mathrm{bps} / \mathrm{Hz}$.

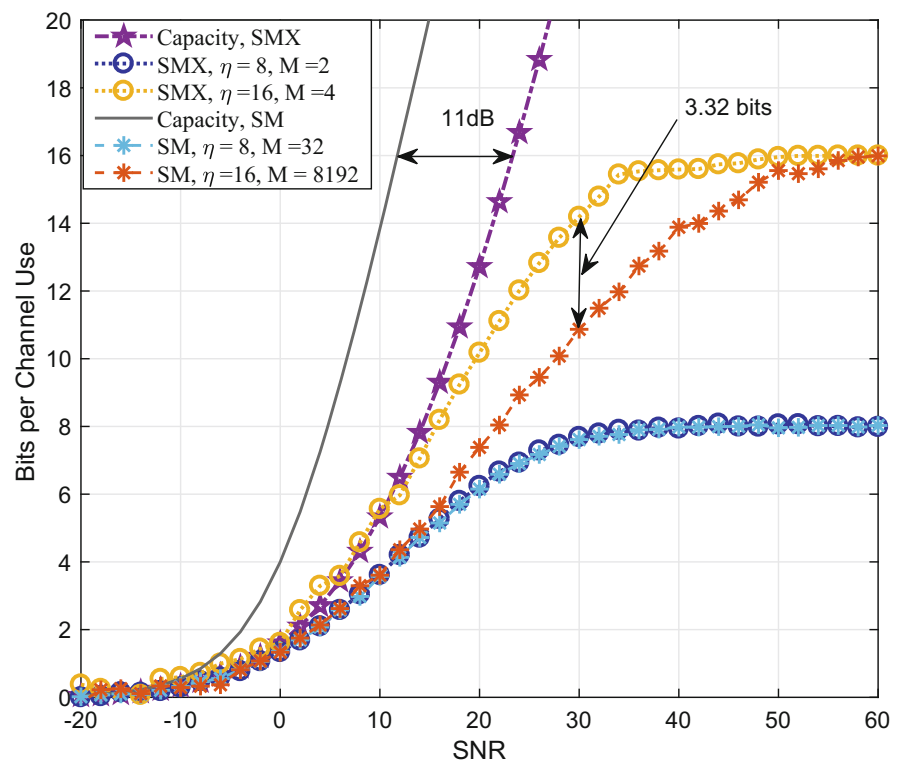

Fig. 3. Mutual information of SM and SMX for variant $\eta$ while assuming $N_{t}=8$ and $N_{r}=4$.

\subsection{Energy Efficiency}

The EE of both systems is studied with respect to the ergodic capacity and for different antenna setups and results are shown in Fig. 4. We define the EE as the ratio between the total number correctly received bits $(C)$ to the total power consumption $\left(P_{s}\right)[18], E E=\frac{C_{\text {ergodic }}}{P_{s}}$. For comparison analysis, we consider 


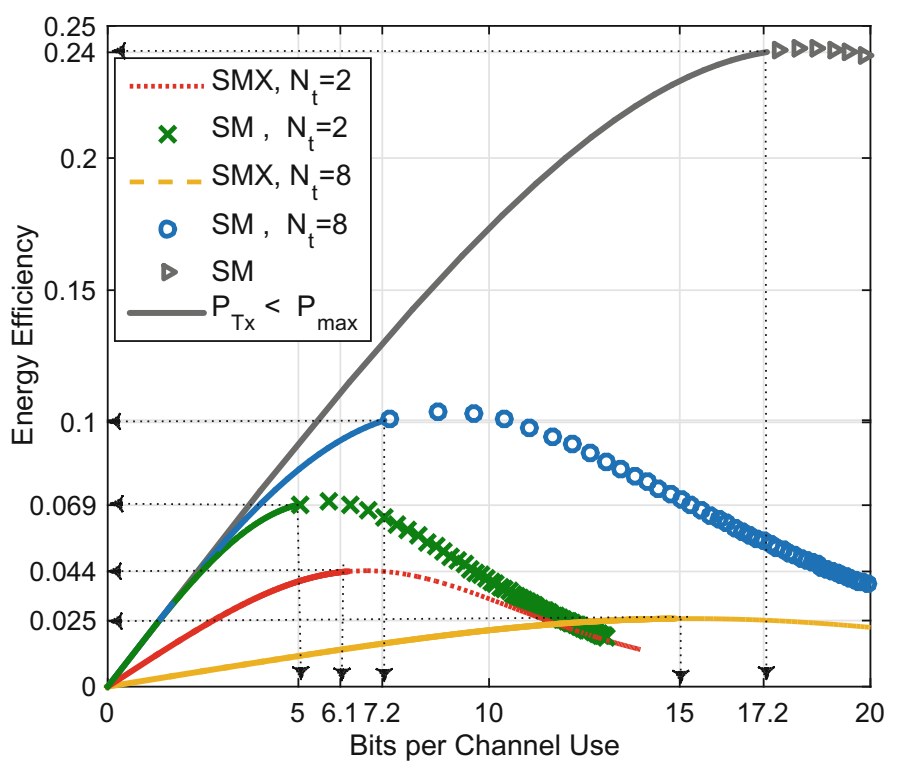

Fig. 4. EE of SM and SMX with $N_{r}=4$, (Solid line) when $\left(P_{T x} \leqslant P_{\max }\right)$.

the EARTH power model, which describes the relation between the total power supplied and the radio frequency (RF) transmit power as [4], $P_{s}=N_{\mathrm{RF}} P_{\min }+$ $m P_{\mathrm{Tx}}$, where $N_{\mathrm{RF}}$ is the required number of RF chains, $N_{\mathrm{RF}}=N_{t}$ for SMX and $N_{\mathrm{RF}}=1$ for $\mathrm{SM}, P_{\min }$ is the minimum RF chain power consumption, $m$ denotes the slope of the load dependent power consumption, and $P_{\mathrm{Tx}}$ is the total RF transmit power. The measurements in [16] were carried in a microcell environment. From [4] for a microcell environment $P_{\min }=53 \mathrm{w}, m=3.1$, and the maximum transmit power per $\mathrm{RF}$ chain is $P_{\max }=6.3 \mathrm{w}$. The figure shows that SM offers better EE than SMX, where SM offers an improvement in the EE by up to $36 \%$ and $74 \%$ compared to SMX for $N_{t}=2$ and $N_{t}=8$, respectively. This is because for SM, the total RF transmit power is fixed. However, for SMX it increases by the number of transmit antennas.

\section{Conclusions}

Two promising MIMO techniques are studied and analyzed in this paper for mmWave communication systems. namely, SM and SMX MIMO systems are evaluated in terms of error probability, capacity and energy efficiency. Reported results reveal that the performance of both systems highly depends on the considered MIMO setup. For the same hardware configuration, SMX is superior to SM since it requires smaller constellation diagram to achieve the same data rate. Yet, SM performance significantly enhances as the number of transmit antennas increase. Considering SM with LS MIMO configuration is very feasible since it can implemented with single RF chain and it does not scale the consumed power. Also, the high frequency of mmWave signals allow for integrating large number of antennas in small dimension without causing significant correlation 
among them. A clear answer to the raised question in the paper title is shown to be equivocal, where both systems can be traded off in terms of performance and energy consumption.

\section{References}

1. 3GPP: Spatial Channel Model for Multiple Input Multiple Output MIMO Simulations. document 3GPP TR 25.996 V12.0.0, September 2014

2. Althunibat, S., Mesleh, R.: Enhancing spatial modulation system performance through signal space diversity. IEEE Commun. Lett. 22(6), 1136-1139 (2018)

3. Andrews, J., et al.: What will 5G be? IEEE J. Sel. Areas Commun. 32(6), 1065$1082(2014)$

4. Auer, G., et al.: How much energy is needed to run a wireless network? IEEE Wirel. Commun. 18(5), 40-49 (2011)

5. Badarneh, O.S., Mesleh, R.: A comprehensive framework for quadrature spatial modulation in generalized fading scenarios. IEEE Trans. Commun. 64(7), 29612970 (2016)

6. Barreto, A., et al.: 5G - wireless communications for 2020. J. Commun. Inf. Syst. 31, 146-163 (2016)

7. Basar, E.: Index modulation techniques for $5 \mathrm{G}$ wireless networks. IEEE Commun. Mag. 54(7), 168-175 (2016)

8. Cisco Visual Networking Index: Global Mobile Data Traffic Forecast Update, 20162021. White Paper, CISCO, February 2017

9. Foschini, G.J.: Layered space-time architecture for wireless communication in a fading environment when using multi-element antennas. Bell Labs Tech. J. 1(2), 41-59 (1996)

10. Kühn, V.: Wireless Communications over MIMO Channels. Wiley, Chichester (2006)

11. Mesleh, R., Haas, H., Sinanović, S., Ahn, C.W., Yun, S.: Spatial modulation. IEEE Trans. Veh. Tech. 57(4), 2228-2241 (2008)

12. Mesleh, R., Hiari, O., Younis, A., Alouneh, S.: Transmitter design and hardware considerations for different space modulation techniques. IEEE Trans. Wirel. Commun. 16(11), 7512-7522 (2017)

13. Mietzner, J., Schober, R., Lampe, L., Gerstacker, W.H., Höeher, P.A.: Multipleantenna techniques for wireless communications - a comprehensive literature survey. IEEE Commun. Surv. Tutor. 11(2), 87-105 (2009)

14. Molisch, A.F., Steinbauer, M., Toeltsch, M., Bonek, E., Thoma, R.S.: Capacity of MIMO systems based on measured wireless channels. IEEE J. Sel. Areas Commun. 20(3), 561-569 (2002)

15. Saleh, A.A.M., Valenzuela, R.: A statistical model for indoor multipath propagation. IEEE J. Sel. Areas Commun. 5(2), 128-137 (1987)

16. Samimi, M.K., Rappaport, T.S.: 3-D millimeter-wave statistical channel model for 5G wireless system design. IEEE Trans. Microw. Theor. Tech. 64(7), 2207-2225 (2016)

17. Shannon, C.: A mathematical theory of communication. Bell Syst. Tech. J. 27, 379-423 \& 623-656 (1948)

18. Stavridis, A., Sinanović, S., Renzo, M.D., Haas, H.: Energy evaluation of spatial modulation at a multi-antenna base station. In: Proceedings of the 78th IEEE Vehicular Technology Conference (VTC), Las Vegas, 2-5 September 2013

19. Zheng, K., Zhao, L., Mei, J., Shao, B., Xiang, W., Hanzo, L.: Survey of large-scale MIMO systems. IEEE Commun. Surv. Tutor. 17(3), 1738-1760 (2015) 